\title{
AUTOMATIC CLASSIFICATION OF BRIDGES AND CONTINENTAL WATER BODIES FROM 3D POINT CLOUDS (AERIAL LiDAR)
}

\author{
Sara Lorite Martínez ${ }^{1}$, Jesús Moreno Jabatoํ, \\ Jesús María Garrido Sáenz de Tejada ${ }^{1}$, Borja Rodríguez-Cuenca ${ }^{1}$ \\ ${ }^{1}$ Instituto Geográfico Nacional, Calle del General Ibáñez de Ibero, 3, 28003, Madrid (Spain) \\ slorite@fomento.es; jmjabato@fomento.es; jmgarrido@fomento.es; brcuenca@fomento.es
}

KEY WORDS: 3D Point Cloud, LiDAR, pattern recognition, automatic classification, hydrography, bridges.

\begin{abstract}
:
The use of algorithms for automatic classification of aerial laser scanner 3D Point Clouds is the main process that improves its thematic quality. The main objectives of using 3D Point Clouds are the description of the surface and the detection of objects. The aim of this proposal for bridge and water detection algorithms is to increase the range and accuracy of the classification parameters of these products obtained with LiDAR technologies. With this methodology, the Digital Elevation Models (DEM) quality is improved and they are obtained by automated models of bridges and hydrography.

This paper describes a methodology to detect and classify bridges and continental water bodies in points using the properties of LiDAR technology such as radiometric and geometric variables implementing indexes like NDVI, NDWI or NFC. In addition, the Network of Roads and Hydrographic models in Spain are used to reduce the area of interest and errors. Part of the province of Teruel (Spain) has been used as study area.
\end{abstract}

\section{INTRODUCTION}

Spanish National Geographic Institute manages and coordinates the PNOA-LiDAR project. The aim of this project is to measure the whole Spanish territory by Aerial Laser Scanner (ALS) every 6 years.

This project started in 2008 and in 2015 the whole national coverage was completed, called First Coverage. Currently, the Second Coverage is being acquired improving the capabilities of the project. The mean density of the registered 3D Point Clouds is around 1 point $/ \mathrm{m}^{2}$, with a RMSE in $\mathrm{z}$ of $15-20 \mathrm{~cm}$. PNOA-LiDAR is an open data project, with free use and distribution licence. Data generated in the PNOA-LiDAR project have been used in research works (Montealegre-Gracia, A. L., 2017), generation of DEMs, Hydrographic models or archaeological discoveries (Fernández-Lozano, J., 2014).

Point Cloud density defines Digital Terrain Model (DTM) cell size, nowadays a 5 meters grid is published for the First Coverage and a 2 meters grid will be published for the Second Coverage.

Another important improvement between the first and the second coverage is the requirement of a simultaneous image registration with near infrared information in addition to RGB which is used to colour the Point Cloud.

\begin{tabular}{|c|c|c|c|}
\hline $\begin{array}{c}\text { Point cloud } \\
\text { density }\end{array}$ & $\begin{array}{c}\text { Point cloud } \\
\text { RMSEz }\end{array}$ & $\begin{array}{c}\text { DTM } \\
\text { RMSEz }\end{array}$ & $\begin{array}{c}\text { DTM } \\
\text { resolution }\end{array}$ \\
\hline $1 \mathrm{p} / \mathrm{m}^{2}$ & $\leq 0.15 \mathrm{~m}$ & $\leq 0.5 \mathrm{~m}$ & $2 \mathrm{~m} \mathrm{X} 2 \mathrm{~m}$ \\
\hline
\end{tabular}

Table 1 - PNOA-LIDAR main technical specifications

For the production of the DTM a manual edition was needed for bridges and water bodies. The goal is to obtain a solution for detecting these elements in an automatic way improving other studies presented before (Zheng, Yi., 2013).

\section{METHODOLOGY}

Automatically classified 3D Point Clouds with R-G-B-NIR values of simultaneous registered images, in LAS format, corresponding to PNOA-LiDAR Project are used as input.

In a first step of the automatic classification each point from the 3D Point Cloud is assigned to one of the following 6 classes, in correspondence with ASPRS specifications (American Society for Photogrammetry \& Remote Sensing, 2010). Class 12 is used as overlap between flight strips in PNOA-LiDAR:

\begin{tabular}{|l|l|}
\hline ID & Class \\
\hline 2 & Ground \\
\hline 3 & Low Vegetation \\
\hline 4 & Medium Vegetation \\
\hline 5 & High Vegetation \\
\hline 6 & Buildings \\
\hline 7 & Noise \\
\hline
\end{tabular}

Table 2 - Classes defined by ASPRS used in PNOA-LiDAR project

This first approximated classification is done using commercial Point Cloud processing software TerraScan (TerraSolid Team, 2018). After trying different strategies, it was concluded that the results obtained in the automatic classification were of sufficient quality to continue to the following phase of the procedure. In addition, the processing time of the automatic classification was optimal with this option (approximately 4 minutes per $2 \times 2 \mathrm{~km}$ file). This automatic classification is only based on geometric features of the point cloud and additional information as the number of returns, or the flight strip numbers. 
Increasing the range of classes and the classification accuracy are the objectives on a second step. For this, other products are combined with the 3D Point Clouds in the classification algorithms to help to reduce processing times, errors and validate them in subsequent processes.

The implementation and execution of the following algorithms is done using Extract Transform and Load (ETL) software FME (Safe Software Team, 2018).

Summarizing, the workflow of the PNOA-LiDAR project is as follows:

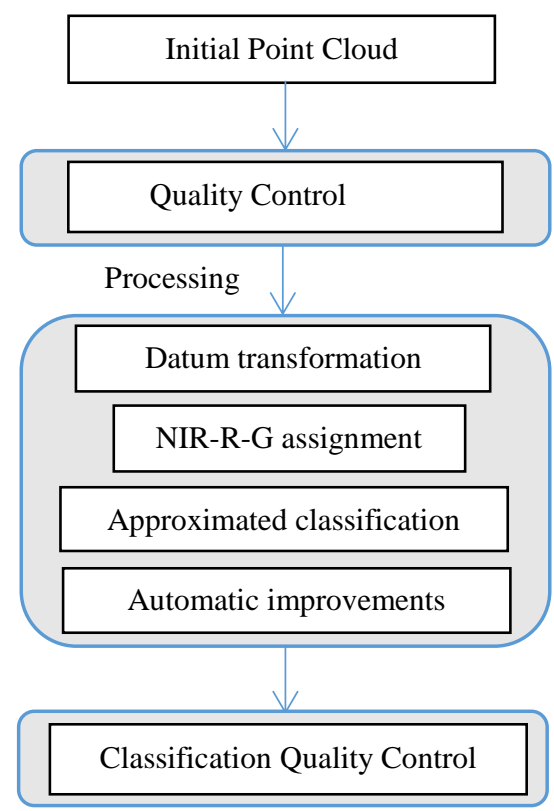

Figure 1 - PNOA-LiDAR Point Clouds processing flow chart

\subsection{Bridge classification}

In the approximated classification the bridge elements are not labelled as Class 17-bridge mainly because nowadays there is no software that provides a good performance automatically. In consequence, these elements are usually misclassified as ground or building points in the approximated classification. This error makes distortions in DTMs or orthophotos.

Using other geometric products is the key of success in the implementation of new classification algorithms. The National Geographic Institute of Spain publishes, as open data policies, the Network of Roads of all Spain in lineal vector format Regarding the bridge detection problem, this product is useful to locate those areas of the point cloud that represent a bridge because this model distinguishes which road sections are elevated using an attribute for this. The proposed method to detect bridges in the point cloud consists of four consecutive steps, as it can be seen in the flow chart shown in Figure 2.

In the first step two polygons vector products are generated, these two products are filtered using the attribute which indicates that the road is elevated. The first product is generated with a 2 meters buffer operator (resulting in a longitudinal polygon, in accordance with the road direction). The second product is generated with different geometric operators resulting perpendicular rectangles to the axis of the road model and dimensions $2 \times 10$ meters (transversal polygons, following the road direction).
In a second step the 3D Point Cloud is compared with vector products generated in the previous procedure. This comparison considers a null hypothesis, all points from the 3D Point Cloud which intersects with both vector products generated are considered Bridge.

In a third step a DEM grid of $2 \times 2$ meters is generated. The boundaries are determined by the second vector product (transversal polygons) generated in the first step and the $\mathrm{Z}$ value is determined by the mean of points selected in the second step.

In a fourth step the original 3D Point Cloud is compared with the DEM generated in step number 3 . If the difference of height is less than 1 meter, those points are considered as Bridge.

New steps are considered as loops from the second and fourth steps where the 3D Point Clouds introduced are the generated in the fourth step.

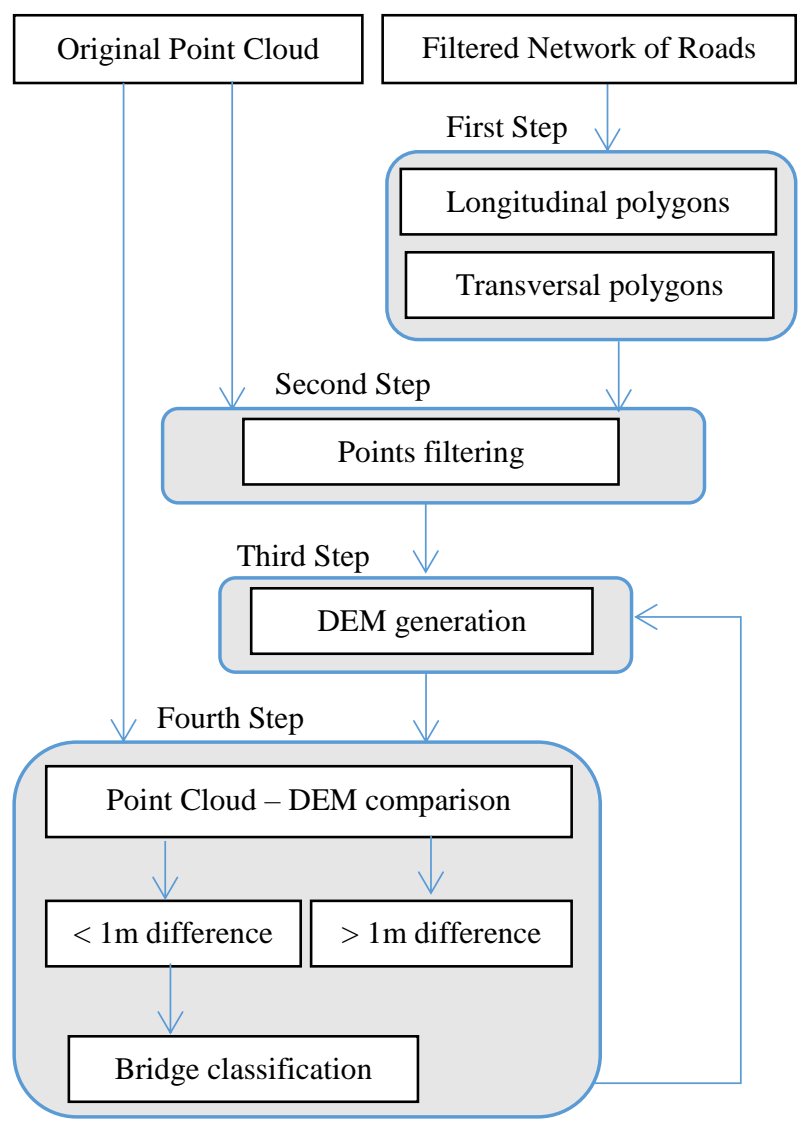

Figure 2 - Bridge classification flow chart

Three loops are implemented to obtain satisfactory results where bridge points are discriminated against ground and building ones.
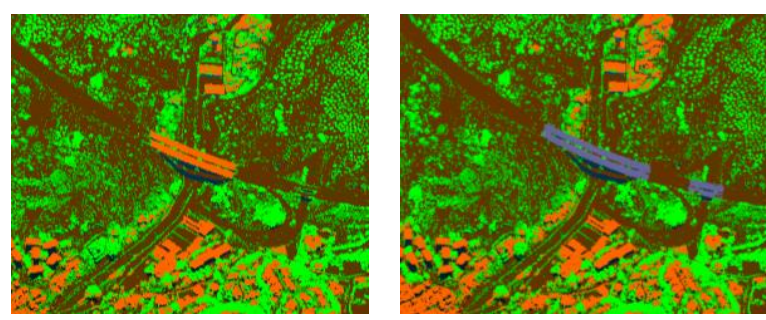

Figure 3 - Comparison between before and after detecting bridge (in grey). Ground points are in brown, building points in orange and vegetation in green 
2.2.

\section{Continental water bodies classification}

Traditionally ALS sensors were not able to detect water, but current systems are able to do it, although no homogeneously. This is a methodology for the classification of those points in water taking into account some previous studies related with the image classification of continental waters (Acharya, Tri D., 2018).

There are different radiometric indexes suitable to carry out jobs of identifying water in an aerial image, i.e. NDVI (Normalized Difference Vegetation Index), NDWI (Normalized Difference Water Index) or Normalized False Colour (NFC):

$$
\begin{gathered}
N D V I=\frac{(N I R-R)}{(N I R+R)} \quad N D W I=\frac{(G-N I R)}{(G+N I R)} \\
\text { Normalized False Colour }=\frac{(N I R * R * G)}{2^{\text {bits }}}
\end{gathered}
$$

They all have identified a similar problem if it is used only the near infrared channel: it is very difficult to distinguish between water and ground shadows. The potential of the proposed method is that combines the potential of radiometric image information with the altitude information of the LiDAR Point Cloud to improve the classification.

In the proposed method firstly a decision tree algorithm is settled implementing 3 conditions for selecting water points using the indexes shown before:

\begin{tabular}{|c|c|c|c|}
\hline Possibilities & NDVI & NDWI & NFC \\
\hline High & $<-0.10$ & $>0.20$ & $>0.03$ \\
\hline High & $<-0.20$ & - & - \\
\hline Medium & $<0.15$ & $(-0.15,0.40)$ & $<0.03$ \\
\hline
\end{tabular}

Table 3 - Radiometric indexes ranges

In addition there are 3 more filters with the aim of avoiding the classification of shadows as water points:

- Approximated classification on first phase: All points which classification is ground or low vegetation are considered, discarding the rest.

- $\quad$ Slope: $Z$ value in points selected as water must not differ on 0,5 meters of the $Z$ mean value in an area of $4 \times 4$ meters. This filter is useful due to the homogeneousness of the $\mathrm{Z}$ value of water points and the existence of big shadows in abrupt areas near to water bodies.

- Hydrographic model: the Hydrography model of reference in Spain is a polygon vector which helps to determine the probability of belonging to a water body. If the convex hull polygon to near points radiometrically selected as water intersects in a percentage of surface greater than $50 \%$ with a polygon of the hydrographic model, those points are considered to have more possibilities to belong to a water body.

The points with higher possibilities are automatically reclassified as class 9 - water and the others, with medium possibilities, are taken into review.

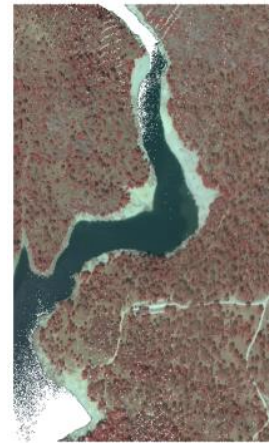

NIR-R-G view

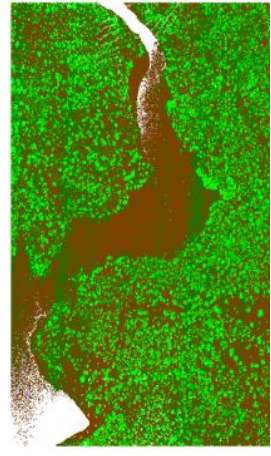

Before Water

Classification

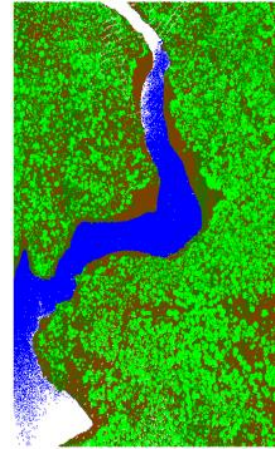

After Water

Classification
Figure 4 - Result example of a water area

During the process of reclassification it is also obtained a polygon layer of the resulting water body contours with an elevation attribute. To digitize the contour it is necessary to erase class 9-water and get the polygon of the hole. This polygon is used later to fill the DTM with a constant $\mathrm{Z}$ in those regions where there is no point cloud information.

\section{RESULTS}

The methodology has been applied in the LiDAR data corresponding to the second national coverage of PNOALIDAR Project in a study area of $1.000 \mathrm{~km}^{2}$ in Teruel province, Spain. This data was collected in 2018. The aforementioned product was generated by the Spanish IGN, with an approximate LiDAR processing time of one month. The area has been chosen because there are sufficient elements and different cases of interest for this work.

For the analysis of the results a visual review has been made, using as ground truth the simultaneous orthophoto obtained with the images registered in the LiDAR flight, in order to identify commission and omission problems and partial detections of bridges and water bodies.

These are the statistical results for the bridge detection procedure (Table 4):

\begin{tabular}{|c|c|c|}
\hline Type of detection & $\begin{array}{c}\text { Number of } \\
\text { bridges }\end{array}$ & Percentage \\
\hline Omissions & 9 & $21 \%$ \\
\hline Commissions & 0 & $0 \%$ \\
\hline Partial detections & 4 & $10 \%$ \\
\hline Full detections & 29 & $69 \%$ \\
\hline Total & 42 & $100 \%$ \\
\hline
\end{tabular}

Table 4 - Bridge results

The total number of points classified as bridge are 18.838 in the whole studied area, achieving an accuracy detection of almost $80 \%$ between full and partial detections.

As an example here are some images of detected situations: 

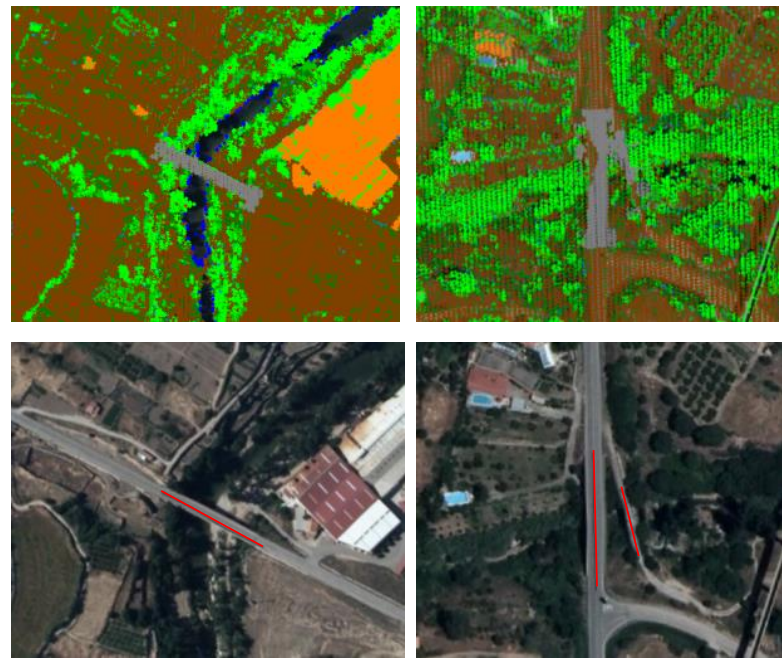

Full detection

Partial detection

Figure 5 - Examples of bridge detection situations (in grey)

Considering the bridge classification results there are some cases where the whole bridge is not classified or points from the ground under the bridge are classified wrongly. In most of these cases the main cause is due to a bad geometry of the lineal road geometries where the axis is not well digitalized along the bridge, so the algorithm is not able of determine which points are elevated.

The process for obtaining the results for water areas has been the same. It is important to know that to analyse the result corresponding to rivers they have been considered as a unique element, with the exception of big water rafts in the middle of the river that have been considered as another element.

These are the statistical results for water bodies extraction (Table 5):

\begin{tabular}{|c|c|c|}
\hline Type of detection & $\begin{array}{c}\text { Number of } \\
\text { water bodies }\end{array}$ & Percentage \\
\hline Omissions & 5 & $16 \%$ \\
\hline Commissions & 2 & $6 \%$ \\
\hline Partial detections & 5 & $16 \%$ \\
\hline Full detections & 20 & $63 \%$ \\
\hline Total & 32 & $100 \%$ \\
\hline
\end{tabular}

Table 5 - Water body results

The total number of points classified as water are 356.168 in the whole studied area. In this case, the detection procedure achieved an accuracy rate of $79 \%$ between full and partial detection.

As an example here there are some images of detected situations:
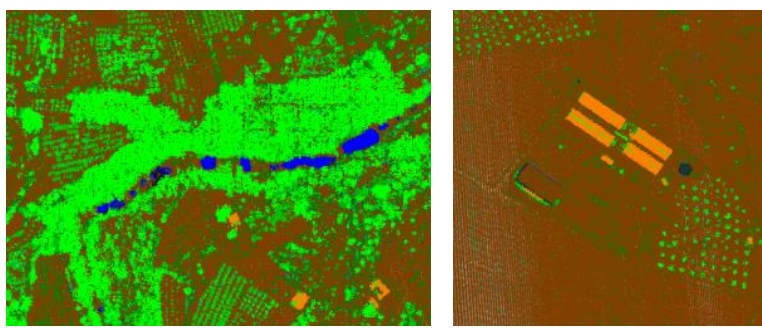

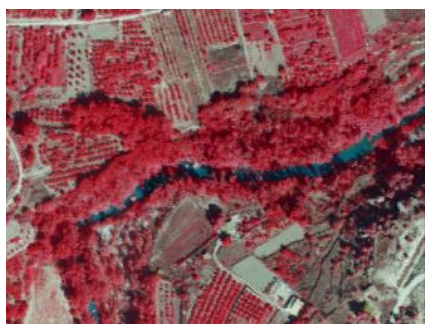

Partial detection

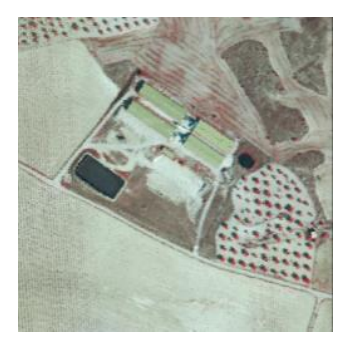

Omission
Figure 6 - Examples of water detection situations (in blue)

Analysing the water results we can say that:

- Commission errors correspond with two shadows in flat areas.

- Partial detections correspond with rivers, due to changes in the water radiometry. The same problem is the cause of the main omissions, encountered in artificial rafts for industrial or agricultural use. When the radiometric of water areas have brightness is also a problem for detection.

\section{CONCLUSIONS}

These procedures show that the objective of bridge and water bodies' detection have been successfully implemented in the study case. Due to this success, it has been introduce as part of the standard production workflow of the PNOA-LiDAR project.

Using other products to establish the area of interest improves the results, but this factor doesn't affect the success of these algorithms without them. The idea is to use this method on one hand, as a first improvement of LiDAR classification, and on the other as an error reporter for the products used. Thus, future results would be more and more accurate thanks to this loop process. It could be also interesting to implement them as tools in a CAD for manual edition.

Image calibration affects to the use of radiometric parameters, so it is necessary a local test to make sure that the tolerances applied are correct in the studied area. Furthermore, as a future work it could be useful the combination of these indexes with LiDAR intensity values.

The application of this method in the whole country with data collected in different years and situations makes the automatization difficult. The algorithms need some parameters of machine learning to improve itself for the processing of the third coverage. 


\section{REFERENCES}

Acharya, Tri D.; Subedi, Anoj; Lee, Dong H. 2018. Evaluation of Water Indices for Surface Water Extraction in a Landsat 8 Scene of Nepal. Sensors 18, no. 8: 2580.

American Society for Photogrammetry \& Remote Sensing. 2010. LAS SPECIFICATION Version 1.4-R6. https://www.asprs.org/wp-content/uploads/2010/12/LAS_14_R6.pdf (17 February 2019).

Fernández-Lozano, J., Gutierrez-Alonso, G. 2014. Aplicación de LiDAR aerotransportado para la cartografía de antiguas labores mineras romanas en el noroeste peninsular. Revista MAPPING. 23. 22-29.

Montealegre-Gracia, A. L., Lamelas-Gracia, M. T., Fernández, R. 2017. Aplicaciones forestales de los datos LiDAR-PNOA en ambiente mediterráneo: su filtrado e interpolación y el modelado de parámetros estructurales con apoyo en trabajo de campo. Doctoral dissertation, Universidad de Zaragoza, Prensas de la Universidad. ISSN 2254-7606

Safe Software Team. 2018. FME software. https://www.safe.com/ (22 March 2019).

Terrasolid Team. 2018. TerraScan - Software for LiDAR Data Processing and 3D Vector Data Creation. http://www.terrasolid.com/products/terrascanpage.php (20 March 2019).

Zheng, Yi. 2013. A Method of Bridge Outline Extraction based on Airborne LiDAR Data. Applied Mechanics and Materials, vol. 303-306, pp. 1048-1055 